\title{
Forum
}

\section{Automatic Radar Plotting Systems}

\author{
A. Harrison
}

FolsowiNg traditional naval tactics when outgunned, Captain Wylie laid a smoke screen in the January issue of the Journal1 and discussed what I 'imply', 'seem to think' and 'suggest'. Before my actual assertion becomes entirely lost to view may I repeat that a prediction of C.P.A., based only on radar data acquired in less than about three minutes, may be seriously in error. Precisely displayed, with no indication of the magnitude of the possible error, it can mislead. To say as he does that this danger is 'supposed' is certainly to beg the question, and supports my belief that the possible size of the error in C.P.A. is still not fully appreciated.

Accepting (for the moment) that the crosses in his Fig. I fairly represent the centres of 'glinting' radar echoes, I quite agree that most observers seeing the eleven crosses would draw a line similar to the hached line. So would I, but a computer without data filtering can operate on the data from two points only, the beginning and the end of the 'plot' separated by a time interval, such as the $30 \mathrm{sec}$ shown. Such a computer, or any technique based only on those two points, will wrongly predict the solid line.

For this kind of glint on ships at a few miles range Tarnowski2 quotes $\pm 0.17^{\circ}$ and Gustafson ${ }^{3} \pm 0.16^{\circ}$ for one standard deviation of the error. So it will often be twice this and sometimes three times, or about $\frac{1}{2}^{\circ}$. Fortunately it is random and can be made negligible by short term filtering $(20 \mathrm{sec})$ as Shuffleton showed. ${ }^{4}$ The program of the above simple computer would have to be extended by this amount to $50 \mathrm{sec}$, and there will be a further delay before the display of a turn or other manœuvre since this smoothing acts to hide it.

Unfortunately Fig. I is quite misleading since it ignores the cyclic errors which are at the root of this discussion. It is fairly well known that marine radar aerials cause an azimuth error when tilted, but the subtlety of this error does not appear to be widely understood. Recently I was privileged to observe an experiment in which the axis of rotation of an aerial was tilted as if subject to ship motion. In the direction of tilt, Bembridge Point at the eastern end of the Isle of Wight was unaffected, but the Nab Tower on a bearing $45^{\circ}$ away appeared to have moved $2^{\circ}$ clockwise on the display. With the aerial axis vertical the $\mathrm{Nab}$ returned to its correct bearing. It may come as a surprise to learn that it repeated its clockwise error when the aerial was tilted away from Bembridge. The reverse tilt does not reverse the error, because as I have shown else- 
where 5 the bearing error (in radians) is ${ }_{4} T^{2} \sin 2 A$ where $T$ is the angle of tilt and $A$ is the target bearing with respect to the direction of tilt. A tilt of $10^{\circ}$ causes a quadrantal error pattern which rises to $\pm 1^{\circ}$ (max).

At sea the angle of tilt depends on wave motion. Draper7 says 'The energy of sea waves is locked in wave components spread over a wide range of wave periods, each of which travels at a speed dictated by its period. Considering the very simple case of a sea with only two wave components, when a crest of one wave overtakes the other, a higher wave will ensue. As a result of this, higher waves come in groups. . . . . This is the reason why it is commonly said that every seventh wave is the highest, although whether it is every fourth or fourteenth depends on the relative speed of the components' and later- '... the duration of about sixty waves, typically about ten minutes'. Summarizing Draper's figures, typical waves have a period of about 10 sec and groups of bigger waves will occur about 40 to $140 \mathrm{sec}$ apart (say one or two minutes) separated by much smaller waves.

A ship in such a sea will tilt with a forced oscillatory motion of similar amplitude sequence and frequency. Thus the magnitude of the error on any particular bearing will depend on two components. The first has a value between zero and some positive maximum; this is approximately of sine squared form. Its period is the second harmonic of roll or pitch (about $5 \mathrm{sec}$ ) and its amplitude rises to a maximum at intervals of one or two minutes, separated by low minima. This is multiplied by the second part, the quadrantal factor $(\sin 2 A)$ which has a value between $+\mathrm{I}$ and $-\mathrm{I}$ including zero on four bearings. The positional error in the target's apparent track is small $\left(\frac{1}{2}^{\circ}\right.$ at 6 miles is only roo yd) but when a short length of this track is used for prediction the effect of this error on C.P.A. may be serious.

Like glint, the rapid variation of this error can be filtered out in $20 \mathrm{sec}$, as Shuffleton showed, leaving a mean value, a bias. This would be negligible if it were constant but it rises from near zero to a peak at intervals of a minute or two, so it cannot be removed by filtering for less than that time as Shuffleton's results also showed. Any system which acquires its data in less than one cycle of error can make its prediction only on the apparent track of that part cycle, which will not usually agree in direction with the real track. The most sophisticated filter is powerless unless it can use data acquired over more than one cycle-about three minutes in this case - or can be told the part of the cycle to which the data refer. An error with similar long and short period components (intercardinal acceleration-sensing) may be produced in the gyro compass output by ship motion effects. This is quadrantal with respect to own ship's heading, being zero on the cardinal headings, but it applies to targets on all bearings. It has been observed by a careful check of the gyro against a distant shore bearing.

The effect of these bearing errors on the predicted C.P.A. can be minimized by plotting (i.e. acquiring data) over a long track. A 6- or I 2- 
minute track, as used in the manual plotting method, would allow a computer without filtering to predict with at least the same accuracy. With filtering, the accuracy can be improved-as Captain Wylie's quotation (4 per cent of range) from R. F. Rigg's informative paper shows; but this C.P.A. error figure is incomplete without the information on page 26 that 'The filtering (tracking) time was fixed at six minutes.' Any attempt to reduce the plot time will increase the C.P.A. error, slowly at first but extremely rapidly when the time becomes less than two 'big wave' cycles-say a minimum of about three minutes, because the filter then becomes ineffective; it is only operating on a part of one cycle of the error and therefore cannot recognize it as an error.

As Grasso shows, 6 one good technique is to use a double filter system, one long ( $6 \mathrm{~min}$ ?) and one short ( $3 \circ \mathrm{sec}$ ?). A prediction is made based on each filter; if they agree within a set margin the long one is trusted. When the target manœuvres the long one lags and they disagree; so the prediction from the short one is used in the full knowledge that errors may be present, but the lag is small, i.e. manœuvres are being displayed quickly but approximately. Later substantial agreement indicates that the target has settled to a new course and speed, and the long term filter can again be trusted. The change-over may be automatic, but the observer should be made aware when predictions of lower accuracy are being displayed.

The aerial error can be removed at source by stabilization, or a vertical reference gyro can feed ship motion to the computer to correct the bear-

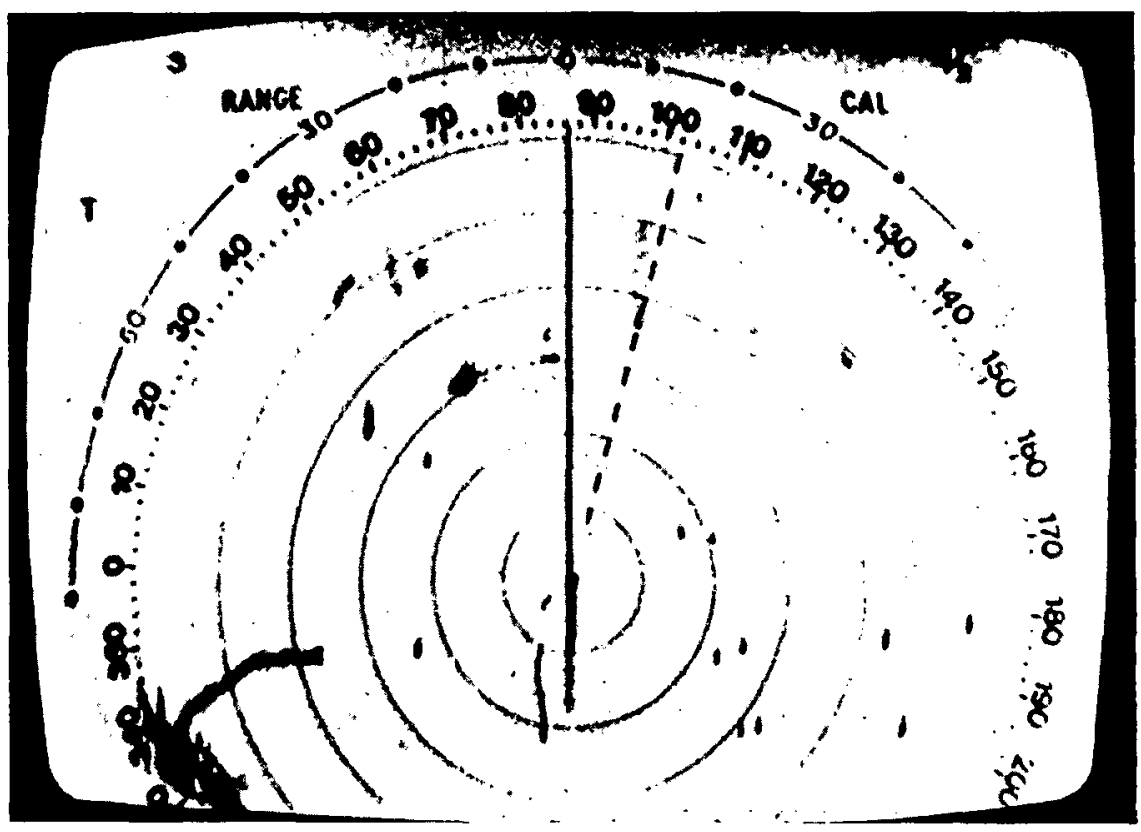

FIG. I 
ing errors. The gyro compass error can be eliminated by fitting it near the roll centre of the ship ${ }^{8}$ or by filtering its output with an aircraft directional gyro.

My comments on these errors do not stand alone. In the discussion following Prior's paper' Watt said 'The fluctuating errors in bearing, apparent under conditions of fairly heavy rolling and pitching on a compass stabilized display, . . . add substantially to the danger (especially if the designer aims to cut short the time between a target's being selected for analysis and the display of computed information) that such a system may display wrong information, with no indication of the unsoundness of the data on which it is based.' In their Collision Avoidance System, Sperry prefer to show the predicted position of a ship not as the apparently precise point indicated by the end of a vector line, but as an oval 'Predicted area of danger'. Presumably they consider it wise to inform the observer of the possible effect of the errors they know to be present. Marshall in his paper ${ }^{10}$ concludes, 'Predictions [of C.P.A.] from radar data can in some cases be in error by several miles'.

So a number of independent sources and my statistical analysis, dismissed by Captain Wylie as 'having no apparent basis', agree that there is an error in C.P.A. caused by bearing errors, although stating it in dif-

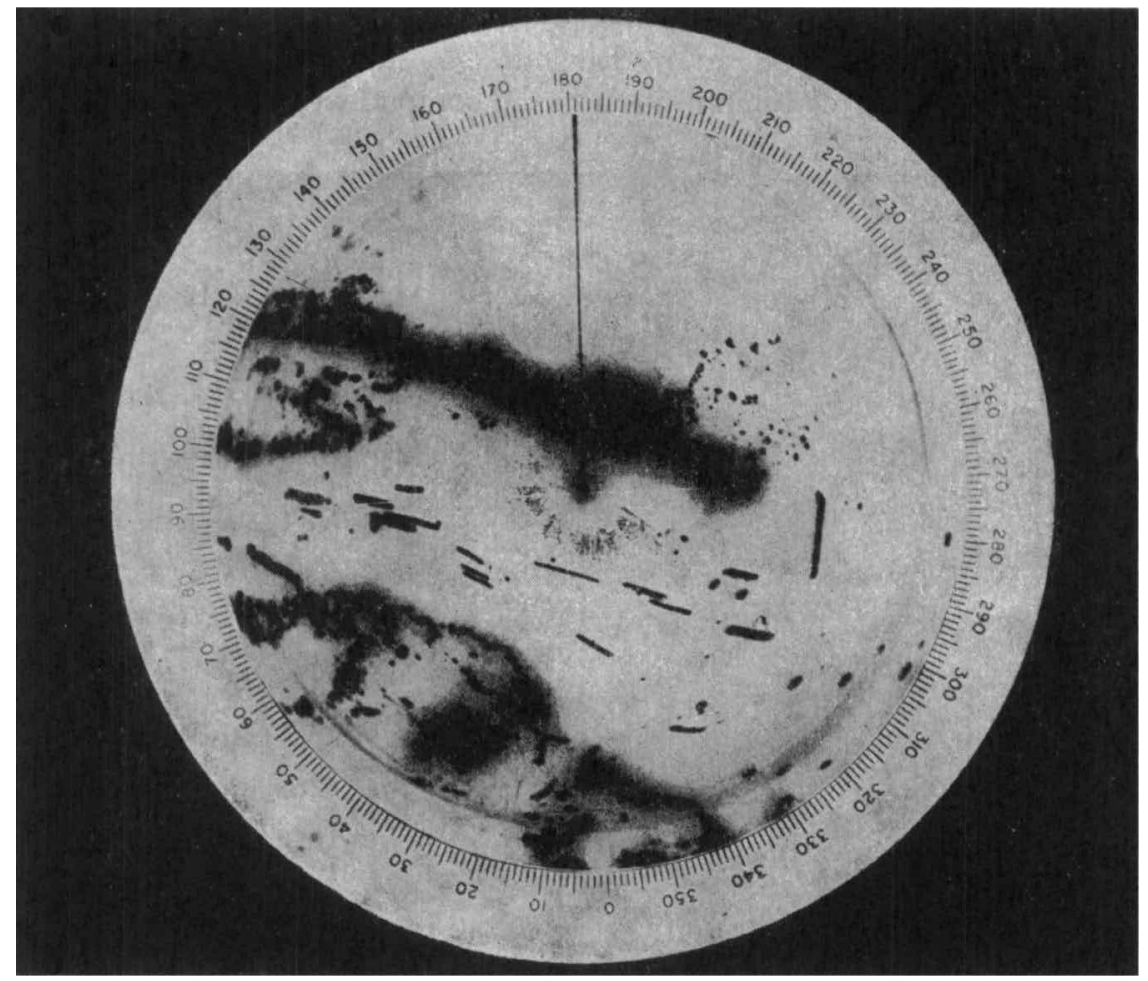

FIG. 2 
ferent ways which are in fact closely equivalent in magnitude. The claim that 'an examination of equipment at present in the field would indicate a significantly better performance' has yet to be proved; experimental evidence to date does not support it and the 'reason to believe that performance will improve' can be no more than a hope, unless the bearing errors are corrected or eliminated at source.

In conclusion, as experimental evidence Fig. I is a photograph of ship tracks, buoys \&cc. in the Thames Estuary, as shown on the 3-mile range of the Kelvin Hughes 'Situation Display'. The buoyed channel one mile to starboard leads to the Medway. The 5 -minute track of an approaching ship on bearing $100^{\circ}$ is broadened by the rapidly changing ( $5 \mathrm{sec}$ ) bearing error and clearly suffers from a cyclic error of about $\pm 1^{\circ}$ with a period of roughly $100 \mathrm{sec}$. This is typical of many examples seen at sea but I have never seen such a track on a land-based radar, e.g. on Southend pier looking at ships in the same area, using Situation Display or Photoplot as in Fig. 2 .

The complete 5-minute track of the ship in Fig. I indicates a C.P.A. of half a mile to starboard in 5 minutes time, just under a mile ahead. The radar data only (raw, filtered, or processed in any way whatsoever) from the first minute of that track (extending from 2.5 to 2.4 miles) would give rise to a prediction which apparently indicates that the other ship will cross our bow somewhere near No. I Sea Reach Buoy, about I.6 miles ahead, and will pass us very closely to port. A precise vector indication of this may well mislead the Master into trying to increase the

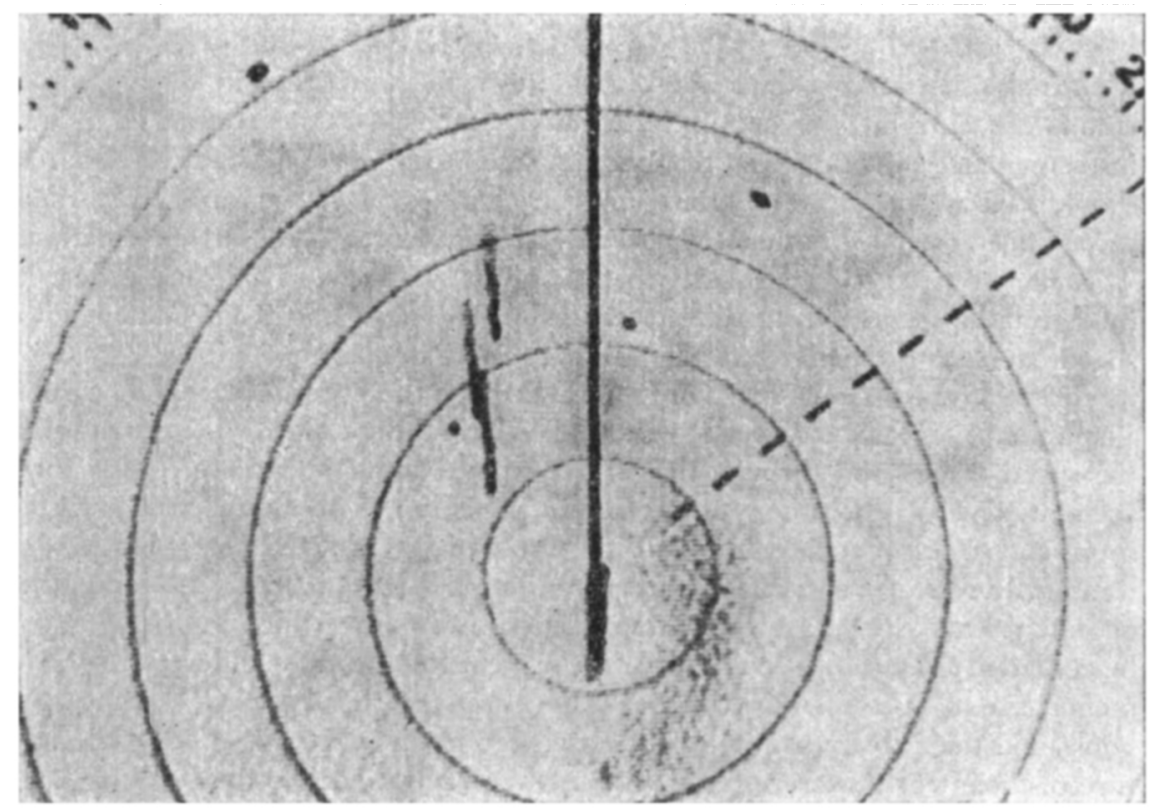

FIG. 3 
clearance by turning to starboard, into the actual track of the other vessel. Is this not Q.E.D. ? Figure 3 is another example, in which three ships on approximately the same bearing appear to yaw simultaneously in formation with a period of 90 sec. Three drunken helmsmen?

\section{REFEREN CES}

1 Herther, J. C. and Wylie, F. J. (1975). This Journal, 28, 97.

2 Tarnowski, P. G. (1964). 'Radar computer for CPA'. ASWE Lab. note TX.64. I.

3 Gustafson, B. G. and $\AA$ s, B. O. (1965). 'System properties of jumping frequency radars', Svenska AB Philips, Stockholm.

4 Shuffleton, W. H. and Evans, H. G. (1972). 'A critical evaluation of an experimental collision avoidance system', International Conference on Advances in Marine Navigational Aids, IEE/IERE, London.

5 Harrison, A. (1972). 'The risk of error in predicted CPA', ibid.

6 Gasparini, O., Grasso, G. and Pardini, S. (1972). 'A smoothing logic with high precision in velocity for naval collision avoidance', $i$ bid.

7 Draper, L. (1971). Severe wave conditions at sea. This Journal, 24, 273.

8 Whipps, S. L. (1971). The advantages to be gained by positioning the master gyro compass unit at the roll centre of a VLCC. Unpub. int. memo., Kelvin Hughes.

9 Prior, P. O. (1973). Situation display. This Journal, 26, 298.

10 Marshall, F. (1973). The accuracy of prediction from primary and secondary radar. This Journal, 26, 282 .

\section{Ship Speed Measurement}

\section{A. Robinson}

If an analysis is made of the speed of a ship using data recorded in log-books the results are extremely disappointing, and do not reflect the accuracies of the instrumentation in use. Even data from a satellite navigator, where the speeds should have an accuracy of better than I per cent, give a $I$-knot band in recorded speed; in a $20-k n o t$ ship this is 5 per cent. Such inaccuracies require explanation.

To begin with one must ask what is meant by ship's speed, and different interpretations come from different people. The navigator is concerned with speed over the ground, while the naval architect or marine engineer is mainly interested in the forward speed of the ship through the water. On the other hand a compiler of ocean current data will require both the speed over the ground and the speed made good through the water, but the latter must take leeway into account. Finally, a weather routing consultant is looking for the actual forward speed the ship can achieve through the water in the prevailing weather conditions. All users of speed data may require other related information, such as the ocean currents and the leeway characteristics of the ship, which can in fact only be compiled from ship speed data. At the present time all these different requirements have to be satisfied by the speeds measured at sea and recorded in the logbooks and their abstracts. Ships' officers compile these records, but by the nature of their work they are biased towards the navigational concept of speed.

Figure I shows the relationship between the different speeds that can be measured at sea. The motive power provided by the engines and transmitted 